\title{
Small amplitude waves in a pre-stressed compressible elas- tic layer with one fixed and one free face
}

\author{
M.I. Lashhab, G.A. Rogerson and L.A. Prikazchikova
}

\begin{abstract}
We address the problem of wave propagation in a pre-stressed elastic layer with mixed boundary conditions, the layer having one fixed the one free face. Numerical analysis provides a good initial insight into the influence of these boundary conditions on dispersion characteristics. In the long wave regime, there is clearly no evidence of low frequency motion and thus an absence of any long wave fundamental mode-like features. In the short wave regime however, the dispersion relations does show evidence of low frequency dispersion phenomena. The first harmonic's short wave phase speed limit is shown to be distinct from that of all other harmonics; this coincides with the associated Rayleigh surface wave speed. The short wave analysis is completed with the derivation of approximate solutions for the higher harmonics. Asymptotic long wave approximations of the dispersion relation are then obtained for motion within the vicinity of the thickness stretch and thickness shear resonance frequencies. These approximations are required to obtain the relative asymptotic orders of the displacement components for frequencies within the vicinity of either the shear or stretch resonance frequencies. This enables an analogue of the asymptotic stress-strainstain to be established through asymptotic integration.
\end{abstract}

Keywords. Wave propagation, pre-stress, compressible material, approximations.

\section{Introduction}

Considerable research effort in recent years has been devoted to elucidating the dynamic response and dispersion characteristics of layered elastic media. The need for such understanding arises through the increasingly exacting demands placed upon structural layered components. One particularly important area of application is the aerospace industry. In addition to this, we remark that multi-layered structures also occur naturally, in some widely differing scenarios. As a specific example, we remark that a layer of coal which is often modelled as a soft elastic layer sandwiched between two much stiffer layers, see [1]. Also, from a mechanical viewpoint, a human artery can be modelled as a three-layered cylindrical structure, see [2].

In this present paper, we aim to contribute to current understanding of layered media, by investigating the dynamic properties of a pre-stressed compressible elastic layered with one fixed and one free face. To facilitate this, the propagation of harmonic waves within this type of structure is investigated. To begin with, a numerical investigation of the of the dispersion relation is carried out. A thorough understanding of the dispersion relation is critical to determining the dynamic response in layered media. Traditionally, fundamental modes have always received considerable attention. For the present problem however, no fundamental-type modes exist over the whole wave number regime. As additional motivation for focussing on the harmonics, we mention the possibly in multi-layered media of a surface wave arising from the cumulative affects of the harmonics, rather than result from a single fundamental mode, see [3]. Such an important feature can easily be overlooked within very delicate numerical techniques. Additionally, in [4] it has alsobeen shown that for certain types of vibrations the 
contributions of higher modes may become significant, with motion within the vicinity of the so-called thickness resonance frequencies having particular applications to fluid-structure interaction. Moreover, some boundary value problems, such as an elastic layer with fixed faces, do not feature fundamental modes at all, see [5] and [6]. For the problem we consider, an additional complication also arises as a consequence of the non-symmetric nature of the problem, resulting in no possible uncoupling into symmetric (extensional) and anti-symmetric (flexural) components.

The problem of dispersion in a pre-stressed elastic plate has seen a number of publications over the last 20 years. After the numerical investigations in the plane strain problem were carried out in [7] and [8], an asymptotic long and short wave analysis was carried out for a variety of layered pre-stressed structures, see [9] and [10]. Three dimensional motion in the form of a plane wave travelling parallel to the plane of the layer was also examined in [11]. Long wave approximations of the dispersion relation have also been used to help derive specific models for long wave motion in pre-stressed elastic plates, see [12] and [13].

This paper is organised as follows. In Section 2 a brief review of the two-dimensional plane strain governing equations is presented, together with the derivation of the dispersion relation. Numerical solutions of the dispersion relation are presented in Section 3 for a Blatz-Ko type of material. Long and short wave asymptotic approximations of the dispersion relation, giving phase speed (or frequency) as an explicit function of wave number for each mode, are obtained in Sections 4 and 5, respectively. The long wave approximations are valid for motion within the vicinity of either the thickness stretch or thickness shear resonance frequencies. In Section 6, these approximations are used to establish the appropriate asymptotic orders of the displacement components in each case. The introduction of non-dimensional spatial and time variable enables asymptotic models to be established through the use of asymptotic integration. Within these one-dimensional models, the long wave displacement field is established in terms of a single function, termed the long wave amplitude. A dispersive governing equation for the long wave amplitude is obtained, for which the dispersion relation is shown to coincide with the long wave approximation obtained from the associated higher dimensional problem.

\section{Basic equations}

In this section we give a brief review of the theory of small time-dependent deformations superimposed upon large static homogeneous deformations. A more detailed account may be found in [14]. We shall consider a body $B$, formed of a homogeneous, non-heat-conducting isotropic elastic material. We suppose that it possesses a natural unstressed state $B_{u}$. A purely homogeneous static deformation is then imposed upon $B_{u}$, resulting in a finitely stressed equilibrium configuration denoted by $B_{e}$. The position vectors of a representative particle are denoted by $\mathbf{X}$ and $\mathbf{x}(\mathbf{X})$ in $B_{u}$ and $B_{e}$, respectively. The deformation from $B_{u}$ to $B_{e}$ is described by the mapping $H$, so that

$$
\mathbf{x}=H(\mathbf{X}), \quad \mathbf{X} \in B_{u}
$$

where $H$ is assumed to be one-to-one and at least twice continuously differentiable. We further consider an incremental time-dependent motion $\mathbf{u}$ superimposed upon $B_{e}$, resulting in the current configuration $B_{t}$. The position vector of a representative particle in $B_{t}$ is denoted by $\tilde{\mathbf{x}}(\mathbf{X}, t)$ and may be expressed as

$$
\tilde{x}_{i}\left(X_{A}, t\right)=x_{i}\left(X_{A}\right)+u_{i}\left(x_{j}, t\right) .
$$

The specific problem to which the above theoretical framework is to be applied is that of harmonic wave propagation in an isotropic, compressible elastic plate of finite thickness $2 h$ and infinite lateral extent. This plate is subjected to a pre-stress in the form of a pure homogeneous strain, defined by the principal stretches $\lambda_{1}, \lambda_{2}$ and $\lambda_{3}$. A Cartesian coordinate system is chosen with axes coincident with the principal axes of deformation in $B_{e}$, oriented such that $O x_{2}$ is normal to the plane of the plate, origin $O$ is in the mid-plane and the upper and lower surfaces located at $x_{2}=h$ and $x_{2}=-h$, respectively. To simplify our analysis, a plane strain assumption is made in which $u_{3} \equiv 0$ and $u_{1}$ and 
$u_{2}$ are independent of $x_{3}$. The two non-trivial equations of motion are given by

$$
\begin{aligned}
& \alpha_{11} u_{1,11}+\gamma_{2} u_{1,22}+\beta u_{2,12}=\rho_{e} \ddot{u}_{1}, \\
& \gamma_{1} u_{2,11}+\alpha_{22} u_{2,22}+\beta u_{1,12}=\rho_{e} \ddot{u}_{2},
\end{aligned}
$$

in which notation from [7] has been exploited, namely

$$
\alpha_{i j}=A_{i i j j}, \quad \gamma_{1}=A_{1212}, \quad \gamma_{2}=A_{2121}, \quad \beta=\alpha_{12}+\gamma_{2}-\sigma_{2},
$$

where $\sigma_{i}, i \in\{1,2\}$ are the principal Cauchy stresses in $B_{e}, \rho_{e}$ is the material density in $B_{e}$ and $A_{i j k l}$ components of the fourth order elasticity tensor. Linearised incremental tractions, associated with a surface having outward unit normal along $O x_{2}$ in $B_{e}$, have components

$$
\tau_{1(2)}=\gamma_{2} u_{1,2}+\left(\gamma_{2}-\sigma_{2}\right) u_{2,1}, \quad \tau_{2(2)}=\alpha_{12} u_{1,1}+\alpha_{22} u_{2,2}
$$

For a plate with traction-free upper boundary and fixed (zero displacement) lower boundary, appropriate boundary conditions are given by

$$
\tau_{1(2)}(h)=\tau_{2(2)}(h)=0, \quad u_{1}(-h)=u_{2}(-h)=0 .
$$

We now seek travelling wave solutions of the equations of motion (3) in the form

$$
\left(u_{1}, u_{2}\right)=\left(U_{1}, U_{2}\right) e^{k q x_{2}} e^{i k\left(x_{1}-v t\right)},
$$

where $k$ is the wave number, $v$ is the phase speed and $q$ is to be determined. After substituting solutions of the form Eq.(7) into the equations of motion (3), a system of linear homogeneous equations is obtained. A non-trivial solution will exist provided the determinant of the system's coefficients is equal to zero, yielding a necessary and sufficient condition, usually referred to as the secular equation, and given by

$$
\alpha_{22} \gamma_{2} q^{4}+\left[\beta^{2}-\alpha_{22}\left(\alpha_{11}-\bar{v}^{2}\right)-\gamma_{2}\left(\gamma_{1}-\bar{v}^{2}\right)\right] q^{2}+\left(\alpha_{11}-\bar{v}^{2}\right)\left(\gamma_{1}-\bar{v}^{2}\right)=0, \quad \bar{v}^{2}=\rho_{e} v^{2} .
$$

Solutions for $u_{1}$ and $u_{2}$ may be represented as a linear combination of the four generally independent solutions associated with the roots of Eq.(8). Substituting these into the boundary conditions results in a homogeneous system of four linear equations in four unknowns. Due to the fact that, unlike the classical free-face problem, the current problem is not symmetric about the mid-plane, the present system can not be decomposed into symmetric (extensional) and antisymmetric (flexural or bending) components. This system possesses non-trivial solutions provided the determinant of its coefficients is equal to zero, yielding the dispersion relation

$$
J_{1}(\bar{v}) \sinh \left(2 q_{1} \eta\right) \sinh \left(2 q_{2} \eta\right)=q_{1} q_{2}\left\{J_{2}(\bar{v})+J_{3}(\bar{v}) \cosh \left(2 q_{1} \eta\right) \cosh \left(2 q_{2} \eta\right)\right\},
$$

where $\eta=k h$ is the scaled wave number and $J_{1}(\bar{v}), J_{2}(\bar{v})$ and $J_{3}(\bar{v})$ are given by

$$
\begin{aligned}
J_{1}(\bar{v})= & {\left[\alpha_{22}\left(\gamma_{2}-\sigma_{2}\right)\left(\alpha_{11}-\bar{v}^{2}\right)+\alpha_{12} \gamma_{2}\left(\gamma_{1}-\bar{v}^{2}\right)\right]^{2} } \\
& -\beta^{2}\left[\alpha_{22}\left(\gamma_{2}-\sigma_{2}\right)^{2}\left(\alpha_{11}-\bar{v}^{2}\right)+\alpha_{12}^{2} \gamma_{2}\left(\gamma_{1}-\bar{v}^{2}\right)-\alpha_{22} \gamma_{2}\left(\alpha_{11}-\bar{v}^{2}\right)\left(\gamma_{1}-\bar{v}^{2}\right)\right], \\
J_{2}(\bar{v})= & 2 \alpha_{22} \gamma_{2} \beta\left\{\left(\gamma_{2}-\sigma_{2}\right)\left[\alpha_{12} \beta-\alpha_{22}\left(\alpha_{11}-\bar{v}^{2}\right)\right]-\alpha_{12} \gamma_{2}\left(\gamma_{1}-\bar{v}^{2}\right)\right\}, \\
J_{3}(\bar{v})= & \alpha_{22} \gamma_{2}\left\{\left[\alpha_{22}\left(\alpha_{11}-\bar{v}^{2}\right)-\gamma_{2}\left(\gamma_{1}-\bar{v}^{2}\right)\right]^{2}\right. \\
& \left.-2 \beta\left[\alpha_{12} \alpha_{22}\left(\alpha_{11}-\bar{v}^{2}\right)+\gamma_{2}\left(\gamma_{2}-\sigma_{2}\right)\left(\gamma_{1}-\bar{v}^{2}\right)\right]+\beta^{2}\left[\beta^{2}-2 \alpha_{12}\left(\gamma_{2}-\sigma_{2}\right)\right]\right\} .
\end{aligned}
$$

To facilitate later establishing of their relative magnitudes within the various long wave regimes, we now express $u_{1}$ and $u_{2}$ in terms of one unknown constant, yielding

$$
\begin{aligned}
& u_{1}=\sum_{n=1}^{2} \frac{i \beta q_{n}}{F\left(q_{n}, \bar{v}\right)}\left[C^{(2 n-1)} \sinh \left(k q_{n} x_{2}\right)+C^{(2 n)} \cosh \left(k q_{n} x_{2}\right)\right] \tilde{C}, \\
& u_{2}=\sum_{n=1}^{2}\left[C^{(2 n-1)} \cosh \left(k q_{n} x_{2}\right)+C^{(2 n)} \sinh \left(k q_{n} x_{2}\right)\right] \tilde{C},
\end{aligned}
$$


where

$$
\begin{aligned}
& C^{(4)}=q_{1} \cosh \left(q_{1} \eta\right) \cosh \left(q_{2} \eta\right) G_{2}\left(q_{1}, \bar{v}\right)\left[G_{1}\left(q_{1}, \bar{v}\right) F\left(q_{2}, \bar{v}\right)-G_{1}\left(q_{2}, \bar{v}\right) F\left(q_{1}, \bar{v}\right)\right] \\
& +q_{2} \sinh \left(q_{1} \eta\right) \sinh \left(q_{2} \eta\right) G_{1}\left(q_{1}, \bar{v}\right) F\left(q_{1}, \bar{v}\right)\left[G_{2}\left(q_{2}, \bar{v}\right)-G_{2}\left(q_{1}, \bar{v}\right)\right], \\
& C^{(3)}=q_{1} \cosh \left(q_{1} \eta\right) \sinh \left(q_{2} \eta\right) G_{2}\left(q_{1}, \bar{v}\right)\left[G_{1}\left(q_{2}, \bar{v}\right) F\left(q_{1}, \bar{v}\right)+G_{1}\left(q_{1}, \bar{v}\right) F\left(q_{2}, \bar{v}\right)\right] \\
& +q_{2} \sinh \left(q_{1} \eta\right) \cosh \left(q_{2} \eta\right) G_{1}\left(q_{1}, \bar{v}\right) F\left(q_{1}, \bar{v}\right)\left[G_{2}\left(q_{1}, \bar{v}\right)+G_{2}\left(q_{2}, \bar{v}\right)\right], \\
& C^{(2)}=\frac{-1}{2 G_{1}\left(q_{1}, \bar{v}\right) F\left(q_{2}, \bar{v}\right) \sinh \left(q_{1} \eta\right)}\left\{C ^ { ( 3 ) } \operatorname { c o s h } ( q _ { 2 } \eta ) \left[G_{1}\left(q_{2}, \bar{v}\right) F\left(q_{1}, \bar{v}\right)\right.\right. \\
& \left.\left.-G_{1}\left(q_{1}, \bar{v}\right) F\left(q_{2}, \bar{v}\right)\right]+C^{(4)} \sinh \left(q_{2} \eta\right)\left[G_{1}\left(q_{2}, \bar{v}\right) F\left(q_{1}, \bar{v}\right)+G_{1}\left(q_{1}, \bar{v}\right) F\left(q_{2}, \bar{v}\right)\right]\right\}, \\
& C^{(1)}=\frac{1}{\cosh \left(q_{1} \eta\right)}\left[C^{(2)} \sinh \left(q_{1} \eta\right)-C^{(3)} \cosh \left(q_{2} \eta\right)+C^{(4)} \sinh \left(q_{2} \eta\right)\right],
\end{aligned}
$$

within which

$$
\begin{aligned}
& F\left(q_{i}, \bar{v}\right)=\alpha_{11}-\bar{v}^{2}-\gamma_{2} q_{i}^{2} \\
& G_{1}\left(q_{i}, \bar{v}\right)=\alpha_{12} \gamma_{2} q_{i}^{2}+\left(\gamma_{2}-\sigma_{2}\right)\left(\alpha_{11}-\bar{v}^{2}\right), \\
& G_{2}\left(q_{i}, \bar{v}\right)=-\alpha_{12} \beta+\alpha_{22}\left(\alpha_{11}-\bar{v}^{2}-\gamma_{2} q_{i}^{2}\right), \quad i \in\{1,2\} .
\end{aligned}
$$

\section{Numerical analysis}

For the purpose of numerical investigation of the dispersion relation (9), a Blatz-Ko strain energy function is employed in the form

$$
W=\frac{\mu}{2}\left(\frac{\lambda_{1}^{2} \lambda_{2}^{2}+\lambda_{1}^{2} \lambda_{3}^{2}+\lambda_{2}^{2} \lambda_{3}^{2}}{\lambda_{1}^{2} \lambda_{2}^{2} \lambda_{3}^{2}}+2 \lambda_{1} \lambda_{2} \lambda_{3}-5\right),
$$

where $\mu$ is the shear modulus. Figures 1(a) and (b) present dispersion curves for a layer with one free and one fixed face. In these figures the scaled phase speed $\bar{v}$ is presented against scaled wave number $\eta$ in respect of the Blatz-Ko strain-energy function Eq.(22). From these plots we first note that there is no fundamental mode-type behaviour within either the long wave regime, nor the long wave limit $\eta \rightarrow 0$. However, in the short wave region, and limit $\eta \rightarrow \infty$, we observe low-frequency (fundamental mode-type) features; note that the short wave limit of the branch with lowest phase speed differs from other harmonics. It will be shown later that this phase speed limit in fact corresponds to the associated Rayleigh surface wave speed. Our numerical investigation also indicates the possibility of wave fronts formed in a variety of ways. We first note the monotonic convergence of the harmonics, to a specific phase speed, as $\eta \rightarrow \infty$. For the parameters used in Figure 1(a), this corresponds to a shear wave with speed $\bar{v}_{S}=\sqrt{\gamma_{1}} \approx 0.62$. Following a labelling system introduced in [10], we refer to a wave front formed this way as a type 1 wave front. We also notice from Figure 1(a) a flattening of the harmonics in a narrow wave speed region, forming a longitudinal wave front, travelling with the speed $\bar{v}_{L}=\sqrt{\alpha_{11}} \approx 1.16$. This wave front is referred to as type 2 .

Figure 1 (b) shows longitudinal and shear wave fronts, both formed as type 2 wave fronts, travelling with speeds $\bar{v}_{L} \approx 0.94$ and $\bar{v}_{S} \approx 0.82$, respectively. The short wave limit of all harmonics, apart from the first, defined by $\bar{v}=\bar{v}_{O} \approx 0.76$, demonstrate oscillatory behaviour in the region below $\bar{v}=\bar{v}_{S}$. We remark that for a pre-stressed compressible elastic material, a shear wave can travel faster, with the same speed or even slower than a longitudinal wave, a fact seemingly first noted in [10]. For investigation of the short wave limiting behaviour we shall use the classification, adopted in 
[10], namely:

$$
\begin{aligned}
\text { Case } 1: & \alpha_{11}>\gamma_{1}, \\
& \text { Subcase } 1(\mathrm{a}): \alpha_{22}\left(\alpha_{11}-\gamma_{1}\right)-\beta^{2}>0 \\
& \text { Subcase } 1(\mathrm{~b}): \alpha_{22}\left(\alpha_{11}-\gamma_{1}\right)-\beta^{2}<0
\end{aligned}
$$

Case $2: \alpha_{11}<\gamma_{1}$,

$$
\begin{aligned}
& \text { Subcase } 2(\mathrm{a}): \gamma_{2}\left(\gamma_{1}-\alpha_{11}\right)-\beta^{2}>0, \\
& \text { Subcase } 2(\mathrm{~b}): \gamma_{2}\left(\gamma_{1}-\alpha_{11}\right)-\beta^{2}<0 .
\end{aligned}
$$

We also present Figure 2, showing scaled frequency $\bar{\omega}$ against scaled wave number $\eta$. From this figure we clearly observe that the first harmonic looks separated from all the others and has its own limit for large $\eta$. As we would expect from previous comments, in Figure 2 all cut-off frequencies are non-zero.

\section{Long wave approximations}

We now investigate motion in the vicinity of the so-called thickness shear and thickness stretch resonance frequencies. The thickness shear resonance frequencies are eigenvalues of the following problem

$$
\gamma_{2} u_{1,22}+\rho_{e} \omega^{2} u_{1}=0
$$

subject to

$$
u_{1,2}=0 \quad \text { at } \quad x_{2}=h, \quad u_{1}=0 \quad \text { at } \quad x_{2}=-h,
$$

see [15]. This problem is obtainable by setting $u_{2}=0$ and $u_{1,1}=0$, in the equations of motion (3), and using the boundary conditions (6) and equations (5), resulting in defining the thickness shear resonance frequencies by

$$
\bar{\omega}^{2}=\gamma_{2} \Lambda_{s h}^{2}, \quad \Lambda_{s h}=\left(n-\frac{1}{2}\right) \frac{\pi}{2}
$$

For thickness stretch resonance, we set $u_{1}=0$ and $u_{2,1}=0$ in Eqs.(3) and (6), to obtain

$$
\alpha_{22} u_{1,22}+\rho_{e} \omega^{2} u_{1}=0
$$

which must be solved subject to

$$
u_{2,2}=0 \quad \text { at } \quad x_{2}=h, \quad u_{2}=0 \quad \text { at } \quad x_{2}=-h,
$$

arriving at the appropriate set of cut-off frequencies, given by

$$
\bar{\omega}^{2}=\gamma_{2} \Lambda_{s t}^{2}, \quad \Lambda_{s t}=\left(n-\frac{1}{2}\right) \frac{\pi}{2 \chi}, \quad \chi=\sqrt{\frac{\gamma_{2}}{\alpha_{22}}},
$$

which define the thickness stretch resonance frequencies. The two families of frequencies presented in Eqs.(25) and (28) are also obtainable by taking the limit $\eta \rightarrow 0$ of the dispersion relation (9). Moreover, equations (13) and (14) may be employed to establish that for thickness shear $u_{1} \sim \eta^{-1} u_{2}$, with thickness stretch characterised by $u_{1} \sim \eta u_{2}$.

In the long wave region, $\bar{v}$ is very large, and in view of the frequency range we consider, we assume that $\bar{v}^{2} / \gamma_{2} \sim \eta^{-2}$. In consequence, within this region both $q_{1}$ and $q_{2}$ are imaginary and may be represented in the form $q_{1}=i \hat{q}_{1}, q_{2}=i \hat{q}_{2}$, with $\hat{q}_{1}$ and $\hat{q}_{2}$ real and positive. The dispersion relation (9) is then expressible as

$$
J_{1}(\bar{v}) \sin \left(2 \hat{q}_{1} \eta\right) \sin \left(2 \hat{q}_{2} \eta\right)=\hat{q}_{1} \hat{q}_{2}\left[J_{2}(\bar{v})+J_{3}(\bar{v}) \cos \left(2 \hat{q}_{1} \eta\right) \cos \left(2 \hat{q}_{2} \eta\right)\right] .
$$

From the definitions of functions $J_{1}(\bar{v})-J_{3}(\bar{v})$, defined in Eq.(10)-Eq.(12), we observe that $J_{1}(\bar{v}) \sim \bar{v}^{4}$, $J_{2}(\bar{v}) \sim \bar{v}^{2}$ and $J_{3}(\bar{v}) \sim \bar{v}^{4}$, with expansions for $\hat{q}_{1}$ and $\hat{q}_{2}$ taking the form

$$
\hat{q}_{1}^{2} \eta^{2}=\frac{\bar{\omega}^{2}}{\alpha_{22}}+Q_{1} \eta^{2}+O\left(\eta^{4}\right), \quad \hat{q}_{2}^{2} \eta^{2}=\frac{\bar{\omega}^{2}}{\gamma_{2}}+Q_{2} \eta^{2}+O\left(\eta^{4}\right),
$$




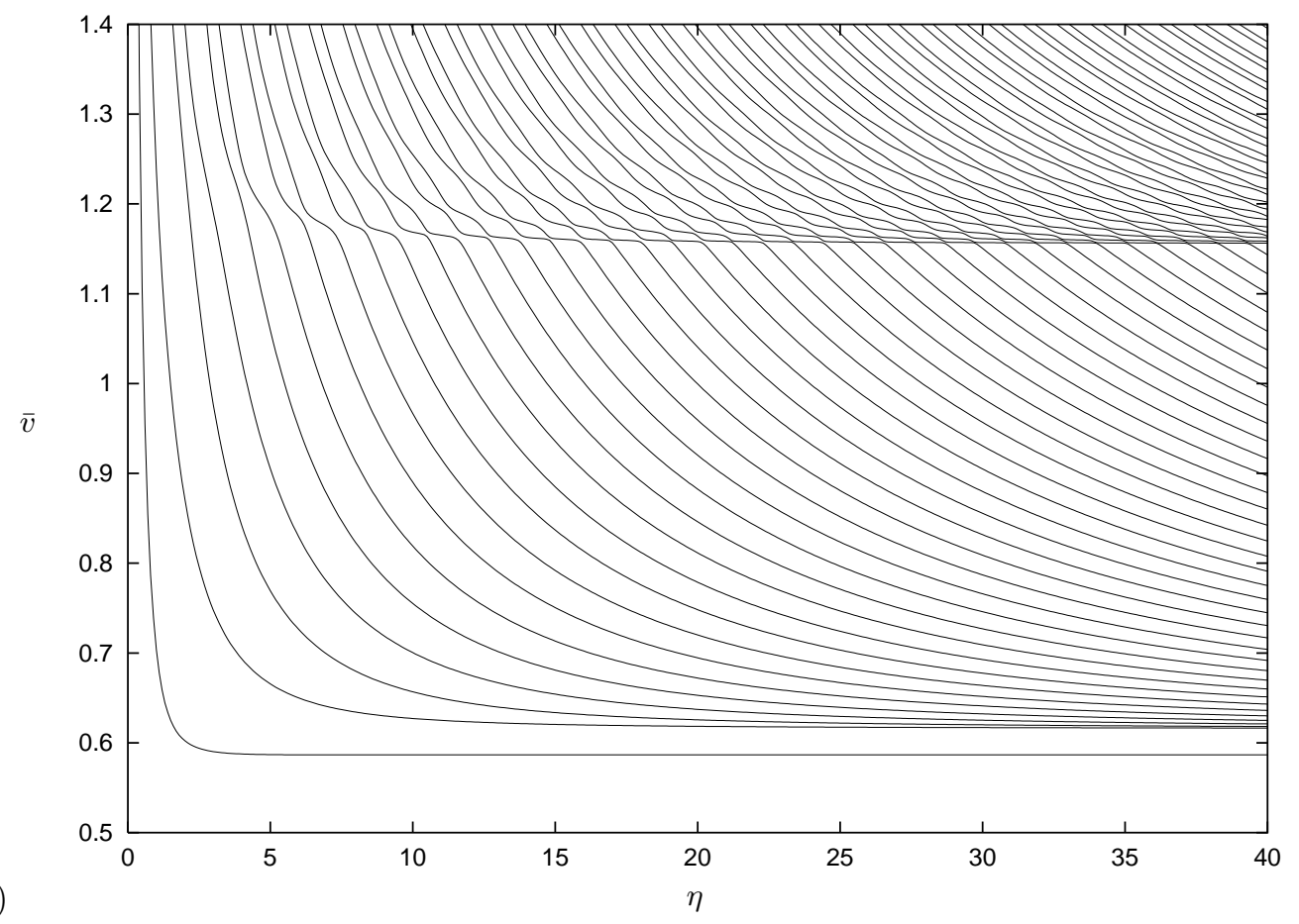

(a)

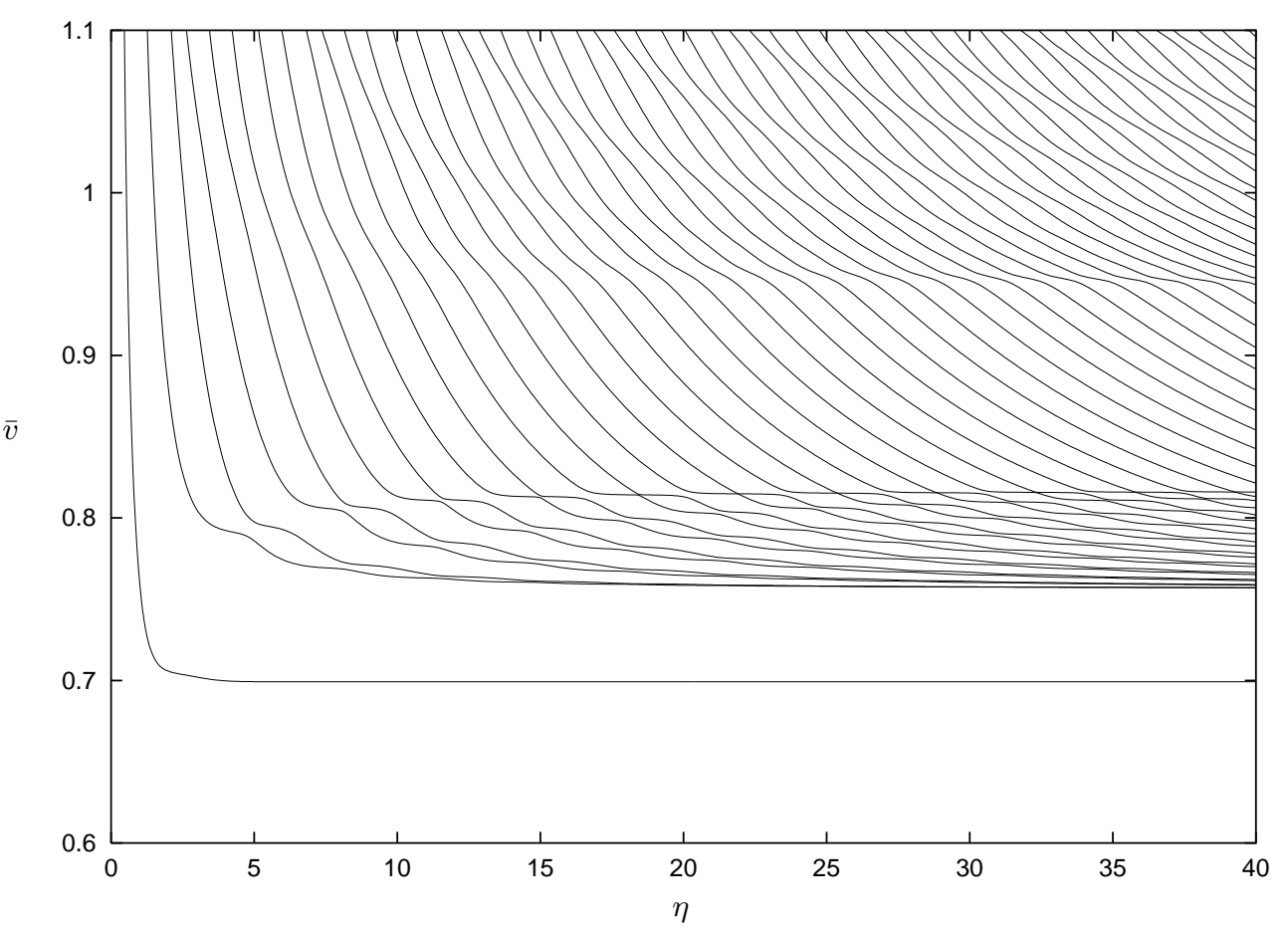

Figure 1. Numerical solution of the dispersion relation for the Blatz-Ko material Eq.(22) with: (a) $\lambda_{1}=1.2, \lambda_{2}=1.3, \lambda_{3}=1.0, \mu=1.0$, corresponding to $\alpha_{11}=$ $1.34, \alpha_{22}=1.14, \alpha_{12}=1.0, \gamma_{1}=0.38, \gamma_{2}=0.45, \sigma_{2}=0.62 ;(b) \lambda_{1}=1.5, \lambda_{2}=$ $1.0, \lambda_{3}=1.0, \mu=1.0$, corresponding to $\alpha_{11}=0.89, \alpha_{22}=2.0, \alpha_{12}=1.0, \gamma_{1}=$ $0.67, \gamma_{2}=0.3, \sigma_{2}=0.33$. 


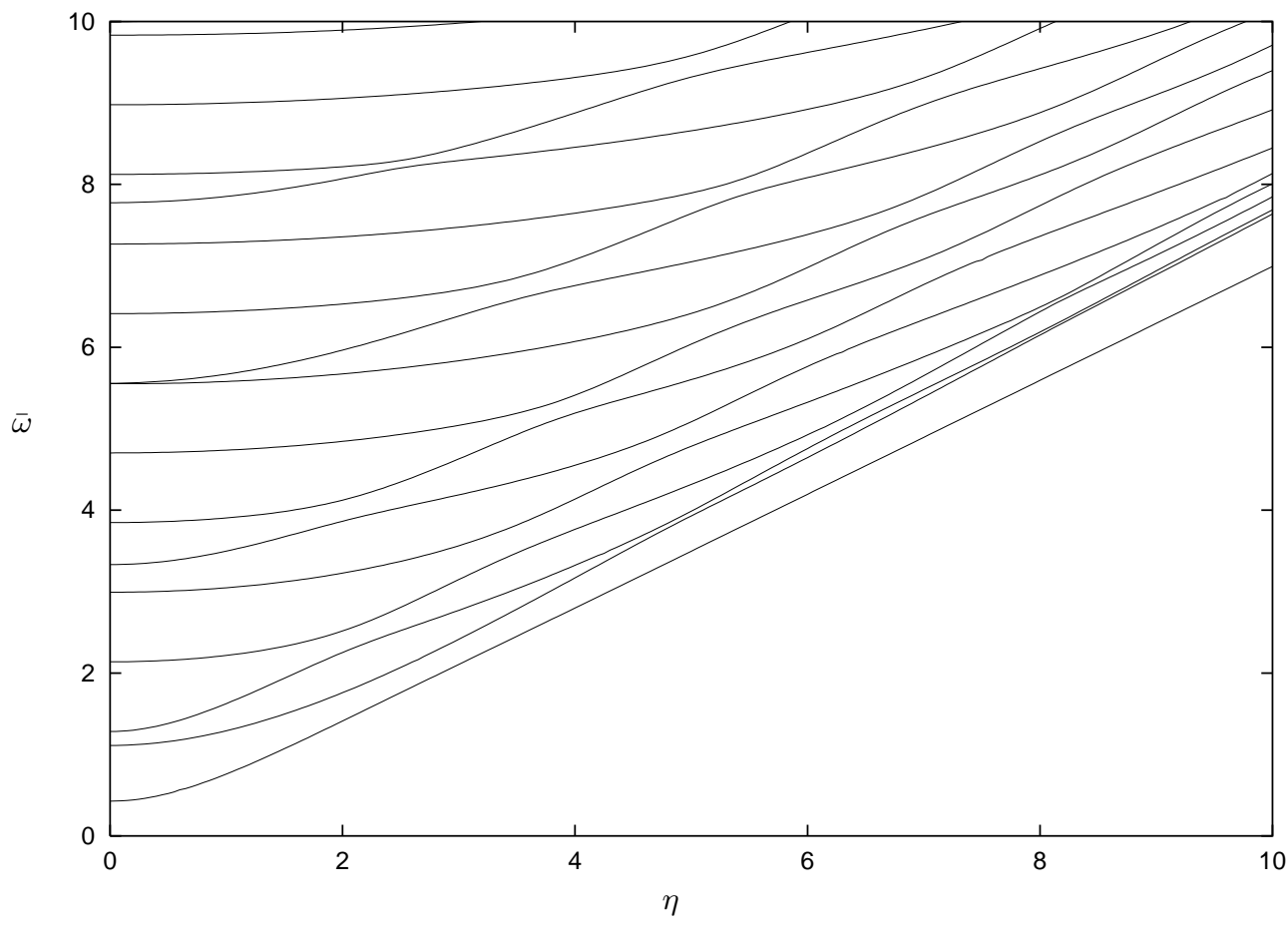

FiguRE 2. Scaled frequency against scaled wave number for a Blatz-Ko material Eq.(22) with the same parameters as in Figure 1(b).

in which $\bar{\omega}=\bar{v} \eta$ and

$$
Q_{1}=\frac{\beta^{2}-\gamma_{1}\left(\gamma_{2}-\alpha_{22}\right)}{\alpha_{22}\left(\gamma_{2}-\alpha_{22}\right)}, \quad Q_{2}=-\frac{\beta^{2}+\alpha_{11}\left(\gamma_{2}-\alpha_{22}\right)}{\gamma_{2}\left(\gamma_{2}-\alpha_{22}\right)}
$$

We assume for the present analysis that $\gamma_{2} \neq \alpha_{22}$. In passing, we remark that if $\gamma_{2}=\alpha_{22}$ there is a coincidence of both the speeds of the longitudinal and shear waves propagating along $O x_{2}$; and the thickness stretch and thickness shear resonance frequencies. For a discussion of the associated dispersion phenomena the reader is referred to [10]. Dividing both sides of the dispersion relation (29) by $\cos \left(2 \hat{q}_{1} \eta\right) \cos \left(2 \hat{q}_{2} \eta\right)$, we obtain two asymptotic balances, namely $\tan \left(2 \hat{q}_{1} \eta\right) \sim O\left(\eta^{-2}\right)$ or $\tan \left(2 \hat{q}_{2} \eta\right) \sim$ $O\left(\eta^{-2}\right)$. In the first case, again using Eq.(30), we obtain at leading order Eq.(28), which corresponds to the motion in the vicinity of the thickness stretch resonance frequencies. Similarly, for $\tan \left(2 \hat{q}_{2} \eta\right) \sim$ $O\left(\eta^{-2}\right)$, using expansion (30), we derive Eq.(25) and thus this case may be interpreted as motion in the vicinity of the thickness shear resonance frequencies. We shall now consider each of these in turn.

\subsection{Asymptotic approximation in the vicinity of the thickness stretch resonance frequencies}

Let us first introduce the notation

$$
K_{2}=\alpha_{22}\left(\gamma_{2}-\sigma_{2}\right)+\alpha_{12} \gamma_{2}, \quad K_{1}=K_{2}^{2}+\alpha_{22} \gamma_{2} \beta^{2}, \quad K_{3}=\left(\alpha_{22}-\gamma_{2}\right)^{2} .
$$

Since $\tan \left(2 \hat{q}_{1} \eta\right) \gg 1$, we may assume that

$$
\hat{q}_{1}=\frac{\chi \Lambda_{s t}}{\eta}+O(\eta), \quad \Lambda_{s t}=\left(n-\frac{1}{2}\right) \frac{\pi}{2 \chi}, \quad n=1,2, \ldots .
$$

At leading order, we obtain

$$
\bar{\omega}^{2}=\gamma_{2} \Lambda_{s t}^{2}+O\left(\eta^{2}\right),
$$


however refining $\hat{q}_{1}$ in the form

$$
\hat{q}_{1}=\frac{\chi \Lambda_{s t}}{\eta}+\phi \eta+O\left(\eta^{3}\right)
$$

results in

$$
\sin \left(2 \hat{q}_{1} \eta\right)=\sin \left(2 \chi \Lambda_{s t}\right)+O\left(\eta^{2}\right), \quad \cos \left(2 \hat{q}_{1} \eta\right)=-2 \phi \sin \left(2 \chi \Lambda_{s t}\right) \eta^{2}+O\left(\eta^{4}\right) .
$$

The asymptotic approximation for the frequency is then readily obtainable in the form

$$
\bar{\omega}^{2}=\gamma_{2} \Lambda_{s t}^{2}+D_{s t} \eta^{2}+O\left(\eta^{4}\right)
$$

where

$$
D_{s t}=\alpha_{22}\left(2 \chi \Lambda_{s t} \phi-Q_{1}\right), \quad \phi=\frac{-2 \gamma_{2} \beta K_{2}+(-1)^{n+1} \chi K_{1} \sin \left(2 \Lambda_{s t}\right)}{(-1)^{n} 2 \gamma_{2}^{2} \Lambda_{s t}^{2} K_{3} \cos \left(2 \Lambda_{s t}\right)},
$$

with $Q_{1}$ defined in Eq.(31).

\subsection{Asymptotic approximation in the vicinity of the thickness shear resonance frequencies}

In the long wave limit we have $\tan \left(2 \hat{q}_{2} \eta\right) \gg 1$, therefore we may assume that

$$
\hat{q_{2}}=\frac{\Lambda_{s h}}{\eta}+\psi \eta+O\left(\eta^{3}\right), \quad \Lambda_{s h}=\left(n-\frac{1}{2}\right) \frac{\pi}{2}
$$

where $\psi$ is to be determined. We then first establish that

$$
\sin \left(2 \hat{q}_{2} \eta\right)=\sin \left(2 \Lambda_{s h}\right)+O\left(\eta^{2}\right), \quad \cos \left(2 \hat{q}_{2} \eta\right)=-2 \psi \sin \left(2 \Lambda_{s h}\right) \eta^{2}+O\left(\eta^{4}\right) .
$$

Substituting all of these approximations into the dispersion relation (29), we deduce that

$$
\psi=\frac{-2 \alpha_{22} \beta \chi K_{2}+(-1)^{n+1} K_{1} \sin \left(2 \Lambda_{s h} \chi\right)}{(-1)^{n} 2 \chi \Lambda_{s h}^{2} \alpha_{22} \gamma_{2} K_{3} \cos \left(2 \Lambda_{s h} \chi\right)} .
$$

Finally, making use of the asymptotic expansion for $\hat{q}_{2}$, given in Eq.(30), we arrive at the following expansion for the scaled frequency

$$
\bar{\omega}^{2}=\gamma_{2} \Lambda_{s h}^{2}+D_{s h} \eta^{2}+O\left(\eta^{4}\right)
$$

where

$$
D_{s h}=\gamma_{2}\left(2 \Lambda_{s h} \psi-Q_{2}\right)
$$

with the definition of $Q_{2}$ given in Eq.(31). Figure 3 demonstrates the accuracy of the obtained asymptotic expansions (37) and (42) for motion in the vicinity of the thickness stretch and shear resonance frequencies, respectively.

\section{Short wave approximations}

Our numerical investigation clearly revealed the existence of low frequency-type motion in the short wave regime in respect of the mode with lowest phase speed. Taking both $q_{1}$ and $q_{2}$ as either real or complex conjugates, and expanding the trigonometric functions in dispersion relation (9) for large $\eta$, we arrive at the short wave limit equation

$$
\sqrt{\alpha_{22} \gamma_{2}} J_{1}(\bar{v})-\sqrt{\left(\alpha_{11}-\bar{v}^{2}\right)\left(\gamma_{1}-\bar{v}^{2}\right)} J_{3}(\bar{v})=0 .
$$

This equation is in fact that associated with the Rayleigh surface wave speed, see [16]. For the material parameters employed within Figures $1(\mathrm{a})$ and (b) the surface wave speeds are given by $\bar{v}_{R} \approx 0.59$ and $\bar{v}_{R} \approx 0.7$, respectively.

We now proceed with the derivation of asymptotic short wave expansions for the other harmonics. Consider first the case when the longitudinal wave speed exceeds that of the shear wave, i.e. $\bar{v}_{L}>\bar{v}_{S}$, and $\alpha_{22}\left(\alpha_{11}-\gamma_{1}\right)-\beta^{2}>0$. In this case, it is readily established that $q_{1}$ is real and $q_{2}$ imaginary in the short wave regime; we thus set $q_{2}=i \hat{q}_{2}$, with $\hat{q}_{2}$ real, positive and tending to zero as $\eta \rightarrow \infty$. The dispersion relation (9) may now be re-cast in the form

$$
J_{1}(\bar{v}) \sinh \left(2 q_{1} \eta\right) \sin \left(2 \hat{q}_{2} \eta\right)=q_{1} \hat{q}_{2}\left[J_{2}(\bar{v})+J_{3}(\bar{v}) \cosh \left(2 q_{1} \eta\right) \cos \left(2 \hat{q}_{2} \eta\right)\right] .
$$






Figure 3. Asymptotic expansions for scaled frequency for a Blatz-Ko material Eq.(22) with the same parameters as in Figure 1(b): dashed lines - in the vicinity of the thickness stretch resonance frequencies, dotted lines - in the vicinity of the thickness shear resonance frequencies.

From the secular equation (8) we obtain an expansion for the scaled wave speed, namely

$$
\bar{v}^{2}=\gamma_{1}+\left(\alpha_{22}-\frac{\beta^{2}}{\alpha_{11}-\gamma_{1}}\right) \hat{q}_{2}^{2}+O\left(\hat{q}_{2}^{4}\right) .
$$

From Eq.(45) we deduce that as $\eta \rightarrow \infty, \sin \left(2 \hat{q}_{2} \eta\right) \sim \hat{q}_{2}$. Hence, we are able to conclude that

$$
\hat{q}_{2}=\frac{\Lambda}{\eta}+\frac{\phi}{\eta^{2}}+O\left(\eta^{-3}\right), \quad \Lambda=\frac{n \pi}{2} .
$$

Therefore, at leading order it is possible to confirm that

$$
\sin \left(2 \hat{q}_{2} \eta\right)=2 \cos (2 \Lambda) \frac{\phi}{\eta}+O\left(\eta^{-2}\right), \quad \cos \left(2 \hat{q}_{2} \eta\right)=\cos (2 \Lambda)+O\left(\eta^{-1}\right)
$$

and

$$
q_{1}=\sqrt{\frac{\alpha_{22}\left(\alpha_{11}-\gamma_{1}\right)-\beta^{2}}{\alpha_{22} \gamma_{2}}} .
$$

Form of dispersion relation presented in Eq.(45), we obtain an expression for $\phi$, given by

$$
\phi=\frac{q_{1} \Lambda J_{3}}{2 J_{1}}
$$

where

$$
\begin{aligned}
& J_{1}=\left[\alpha_{22}\left(\gamma_{2}-\sigma_{2}\right)\left(\alpha_{11}-\gamma_{1}\right)\right]^{2}-\beta^{2} \alpha_{22}\left(\gamma_{2}-\sigma_{2}\right)^{2}\left(\alpha_{11}-\gamma_{1}\right), \\
& J_{3}=\alpha_{22} \gamma_{2}\left\{\alpha_{22}^{2}\left(\alpha_{11}-\gamma_{1}\right)^{2}-2 \beta \alpha_{12} \alpha_{22}\left(\alpha_{11}-\gamma_{1}\right)+\beta^{2}\left[\beta^{2}-2 \alpha_{12}\left(\gamma_{2}-\sigma_{2}\right)\right]\right\} .
\end{aligned}
$$


Finally, we establish the asymptotic expansion for the scaled wave speed in the form

$$
\bar{v}^{2}=\gamma_{1}+\left(\alpha_{22}-\frac{\beta^{2}}{\alpha_{11}-\gamma_{1}}\right)\left(\frac{\Lambda^{2}}{\eta^{2}}+\frac{2 \Lambda \phi}{\eta^{3}}\right)+O\left(\eta^{-4}\right) .
$$

We shall now examine the short wave limit of all harmonics, excluding the first, when the shear wave speed $\bar{v}_{S}$ exceeds the longitudinal wave speed $\bar{v}_{L}$ and the condition $\gamma_{2}\left(\gamma_{1}-\alpha_{11}\right)-\beta^{2}>0$ holds. First, we infer from the secular equation (8) that in the vicinity of the longitudinal (short wave) wave front

$$
\bar{v}^{2}=\alpha_{11}+\left(\gamma_{2}+\frac{\beta^{2}}{\alpha_{11}-\gamma_{1}}\right) \hat{q}_{2}^{2}+O\left(\hat{q}_{2}^{4}\right) .
$$

As $\eta \rightarrow \infty$, the form of dispersion relation presented in Eq.(45) again indicates that $\sin \left(2 \hat{q}_{2} \eta\right) \sim \hat{q}_{2}$. Hence, we may utilise expansions (47) and (48). An expansion for $q_{1}$ at leading order is also obtainable in the form

$$
q_{1}=\sqrt{\frac{\gamma_{2}\left(\gamma_{1}-\alpha_{11}\right)-\beta^{2}}{\alpha_{22} \gamma_{2}}} .
$$

Finally, we arrive at the asymptotic approximation

$$
\bar{v}^{2}=\alpha_{11}+\left(\gamma_{2}+\frac{\beta^{2}}{\alpha_{11}-\gamma_{1}}\right)\left(\frac{\Lambda^{2}}{\eta^{2}}+\frac{2 \Lambda \phi}{\eta^{3}}\right)+O\left(\eta^{-4}\right),
$$

where

$$
\phi=\frac{q_{1} \Lambda \bar{J}_{3}}{2 \bar{J}_{1}}, \quad \Lambda=\frac{n \pi}{2},
$$

and $\bar{J}_{1}, \bar{J}_{3}$ are defined in the form

$$
\begin{aligned}
& \bar{J}_{1}=\left[\alpha_{12} \gamma_{2}\left(\gamma_{1}-\alpha_{11}\right)\right]^{2}-\beta^{2} \alpha_{12}^{2} \gamma_{2}\left(\gamma_{1}-\alpha_{11}\right), \\
& \bar{J}_{3}=\alpha_{22} \gamma_{2}\left\{\gamma_{2}^{2}\left(\gamma_{1}-\alpha_{11}\right)^{2}-2 \beta \gamma_{2}\left(\gamma_{2}-\sigma_{2}\right)\left(\gamma_{1}-\alpha_{11}\right)+\beta^{2}\left[\beta^{2}-2 \alpha_{12}\left(\gamma_{2}-\sigma_{2}\right)\right]\right\} .
\end{aligned}
$$

We present in Figure 4 the scaled phase speed against scaled wave number for the Blatz-Ko strainenergy function Eq.(22). This figure demonstrates good agreement between the numerical solution of the dispersion relation (9) and the short wave approximation (53) in the case for which the short wave limit of the higher harmonics is the associated shear wave speed.

Let us study now the situation when either $\alpha_{22}\left(\alpha_{11}-\gamma_{1}\right)-\beta^{2}<0$, for $\alpha_{11}>\gamma_{1}$; or $\gamma_{2}\left(\gamma_{1}-\alpha_{11}\right)-$ $\beta^{2}<0$, for $\alpha_{11}<\gamma_{1}$. These situations correspond to Subcase 1(b) and Subcase 2(b), respectively. For this case, the short wave limit is $\bar{v}=\bar{v}_{O}$. This involves oscillations of the phase speed as $\eta \rightarrow \infty$, these occurring in the regions $\bar{v}_{O}<\bar{v}<\bar{v}_{S}$ or $\bar{v}_{O}<\bar{v}<\bar{v}_{L}$, for Subcase 1(b) and Subcase 2(b), respectively. In these regions both $q_{1}$ and $q_{2}$ are imaginary, i.e. $q_{1}=i \hat{q}_{1}$ and $q_{2}=i \hat{q}_{2}$, where $\hat{q}_{1}, \hat{q}_{2} \geq 0$ are real and $\hat{q}_{1} \rightarrow \hat{q}_{2}$ as $\eta \rightarrow \infty$. Accordingly, the appropriate short wave behaviour is investigated by setting

$$
\hat{q}_{1}^{2}=a-b, \quad \hat{q}_{2}^{2}=a+b, \quad \text { with } \quad b \rightarrow 0 \quad \text { as } \quad \eta \rightarrow \infty,
$$

in which $a>0, b>0$. It is possible to obtain from Eq.(8) a quadratic for $a$

$$
\begin{aligned}
& \alpha_{22} \gamma_{2}\left(\alpha_{22}-\gamma_{2}\right)^{2} a^{2}+2 \alpha_{22} \gamma_{2}\left[2 \beta^{2}-\left(\alpha_{11}-\gamma_{1}\right)\left(\alpha_{22}-\gamma_{2}\right)\right] a+ \\
& \quad\left[\gamma_{2}\left(\alpha_{11}-\gamma_{1}\right)+\beta^{2}\right]\left[\alpha_{22}\left(\alpha_{11}-\gamma_{1}\right)-\beta^{2}\right]-\alpha_{22} \gamma_{2}\left(\alpha_{22}+\gamma_{2}\right)^{2} b^{2}=0,
\end{aligned}
$$

which for small $b$ implies that

$$
a=a_{0}+a_{1} b^{2}+O\left(b^{4}\right),
$$

where

$$
a_{0}=\frac{1}{\left(\alpha_{22}-\gamma_{2}\right)^{2}}\left[\left(\alpha_{22}+\gamma_{2}\right) \sqrt{\frac{\beta^{2} T_{0}}{\alpha_{22} \gamma_{2}}}-T_{0}-\beta^{2}\right], \quad a_{1}=\frac{\left(\alpha_{22}+\gamma_{2}\right)}{2} \sqrt{\frac{\alpha_{22} \gamma_{2}}{\beta^{2} T_{0}}},
$$

with

$$
T_{0}=\beta^{2}-\left(\gamma_{1}-\alpha_{11}\right)\left(\gamma_{2}-\alpha_{22}\right) \text {. }
$$


Waves in a pre-stressed compressible layer with one fixed and one free face

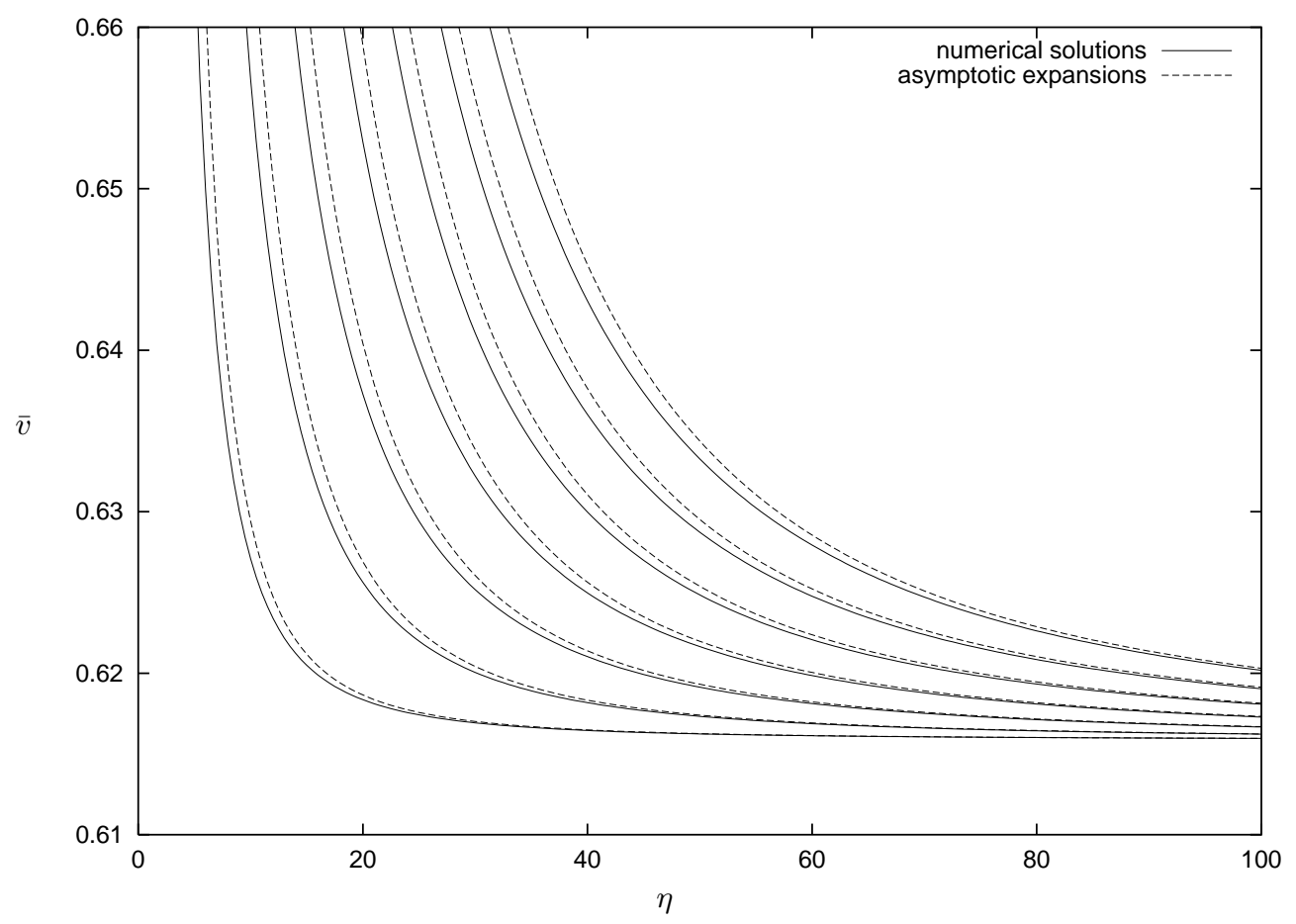

FIGURE 4. Comparison of numerical solution and short wave approximation (53) for the Blatz-Ko material Eq.(22) with the same parameters as in Figure 1(a).

The fact that $a>0$ has been used and it is again assumed that $\gamma_{2} \neq \alpha_{22}$. Note, that in both Subcases $1(\mathrm{~b})$ and 2(b), $T_{0}$ is strictly positive. Then from Eq.(8) we deduce that

$$
\bar{v}^{2}=\bar{v}_{O}^{2}+\frac{1}{\beta} \sqrt{\frac{\alpha_{22}^{3} \gamma_{2}^{3}}{T_{0}}} b^{2}+O\left(b^{4}\right)
$$

where

$$
\bar{v}_{O}^{2}=\frac{\left(\gamma_{2}-\alpha_{22}\right)\left(\gamma_{1} \gamma_{2}-\alpha_{11} \alpha_{22}\right)-\beta^{2}\left(\gamma_{2}+\alpha_{22}\right)+2 \beta \sqrt{\alpha_{22} \gamma_{2} T_{0}}}{\left(\gamma_{2}-\alpha_{22}\right)^{2}} .
$$

Expansions for $\hat{q}_{1}$ and $\hat{q}_{2}$ may now be expressed as

$$
\hat{q}_{1}=\sqrt{a_{0}}\left(1-\frac{b}{2 a_{0}}\right)+O\left(b^{2}\right), \quad \hat{q}_{2}=\sqrt{a_{0}}\left(1+\frac{b}{2 a_{0}}\right)+O\left(b^{2}\right) .
$$

Using expansions Eq.(66) and standard trigonometric formulae, the leading order form of the dispersion relation may be cast into the form

$$
\begin{aligned}
J_{1}\left(\bar{v}_{O}\right)\left(\sin ^{2}\left(2 \sqrt{a_{0}} \eta\right)\right. & \left.-\sin ^{2}\left(\frac{\eta b}{\sqrt{a_{0}}}\right)\right) \\
= & a_{0}\left\{J_{2}\left(\bar{v}_{O}\right)+J_{3}\left(\bar{v}_{O}\right)\left(1-\sin ^{2}\left(2 \sqrt{a_{0}} \eta\right)-\sin ^{2}\left(\frac{\eta b}{\sqrt{a_{0}}}\right)\right)\right\} .
\end{aligned}
$$

An expression for $b$ may be obtained as

$$
b=\frac{B_{0}}{\eta}+O\left(\frac{1}{\eta^{2}}\right)
$$




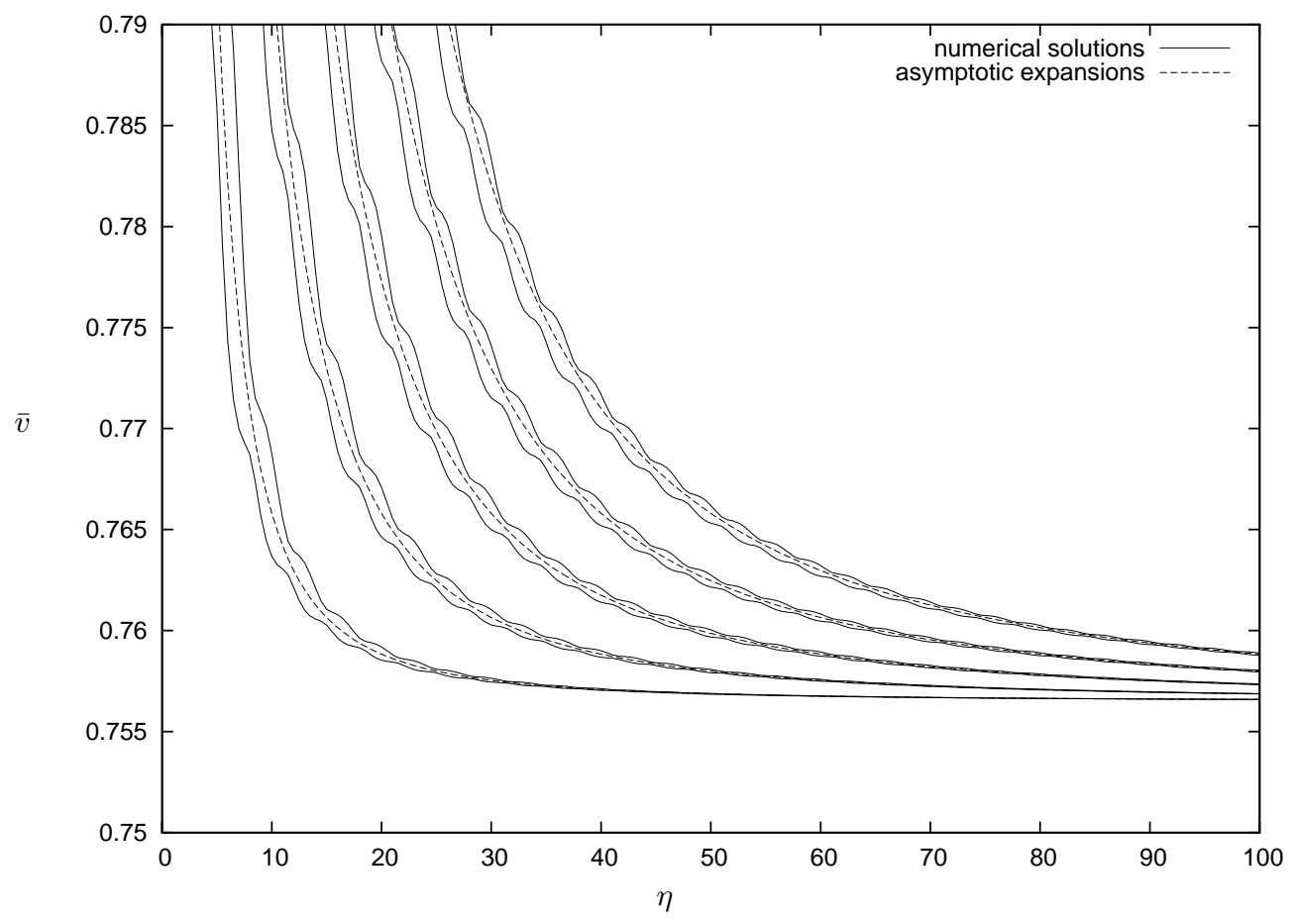

Figure 5. Comparison of numerical solution and short wave approximation Eq.(70) for the Blatz-Ko material Eq.(22) with the same parameters as in Figure 1(b).

where

$$
B_{0}=\frac{\sqrt{a_{0}}}{2}\left\{ \pm \arccos \left(1+\frac{2 \sin ^{2}\left(2 \sqrt{a_{0}} \eta\right)\left(a_{0} J_{3}\left(\bar{v}_{O}\right)+J_{1}\left(\bar{v}_{O}\right)\right)}{a_{0} J_{3}\left(\bar{v}_{O}\right)-J_{1}\left(\bar{v}_{O}\right)}\right)+2 \pi k\right\} .
$$

Finally, we obtain an asymptotic expansion for the phase speed in the form

$$
\bar{v}^{2}=\bar{v}_{O}^{2}+\frac{1}{\beta} \sqrt{\frac{\alpha_{22}^{3} \gamma_{2}^{3}}{T_{0}}} \frac{B_{0}^{2}}{\eta^{2}}+O\left(\frac{1}{\eta^{3}}\right) .
$$

Figure 5 demonstrates good agreement between numerical solution of the dispersion relation and asymptotic expansion Eq.(70). It is worth noting that in this case the coefficient $a_{0} J_{3}\left(\bar{v}_{O}\right)+J_{1}\left(\bar{v}_{O}\right)$ is numerically very close to zero. Two oscillating branches are therefore approximated by what essentially looks like one non-oscillating short wave approximation.

\section{Long wave high frequency models}

Our aim in this section is the derivation of one-dimensional asymptotic models for long wave high frequency motion. We require these models to be consistent with our previous asymptotic analysis of the dispersion relations. Investigation of the relative magnitudes of displacements motivates appropriate re-scalings. After re-casting the equations of motion and boundary conditions in terms of new variables, we derive an appropriate system of governing equations at various orders. One-dimensional asymptotic long wave models for motion in the vicinity of the thickness stretch and shear resonance frequencies, will be derived; we begin with stretch resonance. 


\subsection{Stretch resonance frequencies}

The relative orders of displacements may be established from Eq.(13) and Eq.(14), taking into account expansions Eq.(35)-Eq.(37), revealing that $u_{1} \sim \eta u_{2}$, motivating the re-scaling

$$
u_{1}=\eta l u_{1}^{*}, \quad u_{2}=l u_{2}^{*} .
$$

We also introduce appropriate non-dimensional spatial coordinates and time in the form

$$
x_{1}=l \xi, \quad x_{2}=\ln \zeta, \quad t=\ln \sqrt{\frac{\rho_{e}}{\alpha_{22}}} \tau .
$$

Within the vicinity of the thickness stretch resonance frequencies, we assume that

$$
u_{k, \tau \tau}^{*}+\chi^{2} \Lambda_{s t}^{2} u_{k}^{*} \sim \eta^{2} u_{k}^{*}, \quad k=1,2, \quad \Lambda_{s t}=\left(n-\frac{1}{2}\right) \frac{\pi}{2 \chi}, \quad n=1,2, \ldots .
$$

Within our analysis we will seek approximations for the displacements in the form of the power series $u_{i}=u_{i}^{(0)}+\eta^{2} u_{2}^{(2)}+\ldots$. Substituting the new variables into the equations of motion (3) and the boundary conditions (6), and making use of the above relations, we arrive at the system of equations at various orders

$$
\begin{gathered}
\gamma_{2} u_{1, \zeta \zeta}^{(2 m)}+\gamma_{2} \Lambda_{s t}^{2} u_{1}^{(2 m)}+\beta u_{2, \xi \zeta}^{(2 m)}+\alpha_{11} u_{1, \xi \xi}^{(2 m-2)} \\
-\alpha_{22} \eta^{-2}\left[u_{1, \tau \tau}^{(2 m-2)}+\chi^{2} \Lambda_{s t}^{2} u_{1}^{(2 m-2)}\right]=0, \\
\alpha_{22} u_{2, \zeta \zeta}^{(2 m)}+\gamma_{2} \Lambda_{s t}^{2} u_{2}^{(2 m)}+\beta u_{1, \xi \zeta}^{(2 m-2)}+\gamma_{1} u_{2, \xi \xi}^{(2 m-2)} \\
-\alpha_{22} \eta^{-2}\left[u_{2, \tau \tau}^{(2 m-2)}+\chi^{2} \Lambda_{s t}^{2} u_{2}^{(2 m-2)}\right]=0, \\
\gamma_{2} u_{1, \zeta}^{(2 m)}+\left(\gamma_{2}-\sigma_{2}\right) u_{2, \xi}^{(2 m)}=0 \quad \text { at } \quad \zeta=1, \\
\alpha_{22} u_{2, \zeta}^{(2 m)}+\alpha_{12} u_{1, \xi}^{(2 m-2)}=0 \quad \text { at } \quad \zeta=1, \\
u_{1}^{(2 m)}=u_{2}^{(2 m)}=0 \quad \text { at } \zeta=-1 .
\end{gathered}
$$

Within Eq.(74)-Eq.(78) anything having a negative superscript is assumed to be zero.

6.1.1. Leading order problem. The leading order problem, with $m=0$, is formulated through

$$
\begin{aligned}
& \gamma_{2} u_{1, \zeta \zeta}^{(0)}+\beta u_{2, \xi \zeta}^{(0)}+\gamma_{2} \Lambda_{s t}^{2} u_{1}^{(0)}=0, \\
& u_{2, \zeta \zeta}^{(0)}+\chi^{2} \Lambda_{s t}^{2} u_{2}^{(0)}=0, \\
& \gamma_{2} u_{1, \zeta}^{(0)}+\left(\gamma_{2}-\sigma_{2}\right) u_{2, \xi}^{(0)}=0, \quad u_{2, \zeta}^{(0)}=0 \quad \text { at } \zeta=1, \\
& u_{1}^{(0)}=u_{2}^{(0)}=0 \quad \text { at } \zeta=-1 .
\end{aligned}
$$

The solution of the leading order problem is readily obtained and written in the form

$$
\begin{aligned}
& u_{1}^{(0)}=V_{1}^{(0,0)} \cos \left(\Lambda_{s t} \zeta\right)+U_{1}^{(0,0)} \sin \left(\Lambda_{s t} \zeta\right)+v_{1}^{(0,0)} \cos \left(\chi \Lambda_{s t} \zeta\right)+u_{1}^{(0,0)} \sin \left(\chi \Lambda_{s t} \zeta\right), \\
& u_{2}^{(0)}=v_{2}^{(0,0)}\left(\tan \left(\chi \Lambda_{s t}\right) \sin \left(\chi \Lambda_{s t} \zeta\right)+\cos \left(\chi \Lambda_{s t} \zeta\right)\right)
\end{aligned}
$$

where

$$
\begin{aligned}
V_{1}^{(0,0)} & =\frac{v_{2, \xi}^{(0,0)}}{\chi \Lambda_{s t}\left(\gamma_{2}-\alpha_{22}\right) \cos \left(2 \Lambda_{s t}\right)}\left(\frac{\chi K_{2} \sin \left(\Lambda_{s t}\right)}{\gamma_{2} \cos \left(\chi \Lambda_{s t}\right)}-2 \beta \sin \left(\chi \Lambda_{s t}\right) \cos \left(\Lambda_{s t}\right)\right), \\
U_{1}^{(0,0)} & =\frac{\cos \left(\Lambda_{s t}\right) v_{2, \xi}^{(0,0)}}{\chi \Lambda_{s t}\left(\gamma_{2}-\alpha_{22}\right) \cos \left(2 \Lambda_{s t}\right)}\left(\frac{\chi K_{2}}{\gamma_{2} \cos \left(\chi \Lambda_{s t}\right)}-2 \beta \sin \left(\chi \Lambda_{s t}\right) \tan \left(\Lambda_{s t}\right)\right), \\
v_{1}^{(0,0)} & =-\frac{\beta \tan \left(\chi \Lambda_{s t}\right) v_{2, \xi}^{(0,0)}}{\chi \Lambda_{s t}\left(\alpha_{22}-\gamma_{2}\right)}, \quad u_{1}^{(0,0)}=\frac{\beta v_{2, \xi}^{(0,0)}}{\chi \Lambda_{s t}\left(\alpha_{22}-\gamma_{2}\right)},
\end{aligned}
$$


with $K_{2}$ defined in Eq.(32). The solution for the displacement components have now all been found in terms of the long wave amplitude $v_{2}^{(0,0)}(\xi, \tau)$. A governing equation for $v_{2}^{(0,0)}$ may be found by considering the next order problem.

6.1.2. Second order problem. In order to complete the leading order solution, and derive an equation for $v_{2}^{(0,0)}$, we need only consider the second order equation of motion

$$
\alpha_{22} u_{2, \zeta \zeta}^{(2)}+\gamma_{2} \Lambda_{s t}^{2} u_{2}^{(2)}=\eta^{-2} \alpha_{22}\left(\chi^{2} \Lambda_{s t}^{2} u_{2}^{(0)}+u_{2, \tau \tau}^{(0)}\right)-\gamma_{1} u_{2, \xi \xi}^{(0)}-\beta u_{1, \zeta \xi}^{(0)},
$$

subject to the boundary conditions

$$
\alpha_{22} u_{2, \zeta}^{(2)}+\alpha_{12} u_{1, \xi}^{(0)}=0 \quad \text { at } \quad \zeta=1, \quad u_{2}^{(2)}=0 \quad \text { at } \quad \zeta=-1 .
$$

The solution for $u_{2}^{(2)}$ may be obtained in the form

$$
\begin{array}{r}
u_{2}^{(2)}=u_{2}^{(2,0)} \sin \left(\chi \Lambda_{s t} \zeta\right)+v_{2}^{(2,0)} \cos \left(\chi \Lambda_{s t} \zeta\right)+U_{2}^{(2,0)} \sin \left(\Lambda_{s t} \zeta\right)+V_{2}^{(2,0)} \cos \left(\Lambda_{s t} \zeta\right) \\
+u_{2}^{(2,1)} \zeta \sin \left(\chi \Lambda_{s t} \zeta\right)+v_{2}^{(2,1)} \zeta \cos \left(\chi \Lambda_{s t} \zeta\right),
\end{array}
$$

where

$$
\begin{aligned}
U_{2}^{(2,0)} & =\frac{\beta V_{1, \xi}^{(0,0)}}{\Lambda_{s t}\left(\gamma_{2}-\alpha_{22}\right)}, \quad V_{2}^{(2,0)}=-\frac{\beta U_{1, \xi}^{(0,0)}}{\Lambda_{s t}\left(\gamma_{2}-\alpha_{22}\right)}, \quad v_{2}^{(2,1)}=-\tan \left(\chi \Lambda_{s t}\right) u_{2}^{(2,1)}, \\
u_{2}^{(2,1)} & =\frac{1}{2 \chi \Lambda_{s t}}\left[\eta^{-2}\left(\chi^{2} \Lambda_{s t}^{2} v_{2}^{(0,0)}+v_{2, \tau \tau}^{(0,0)}\right)+Q_{1} v_{2, \xi \xi}^{(0,0)}\right] .
\end{aligned}
$$

The definition of $Q_{1}$ may be found in Eq.(31). We are also able to derive a governing equation for $v_{2}^{(0,0)}$ in the form

$$
D_{s t} v_{2, \xi \xi}^{(0,0)}-\alpha_{22} \eta^{-2}\left(\chi^{2} \Lambda_{s t}^{2} v_{2}^{(0,0)}+v_{2, \tau \tau}^{(0,0)}\right)=0
$$

An alternatively form of this equation, in terms of the original variables $x_{1}$ and $t$ and a new function $v^{[0]}\left(x_{1}, t\right)$, is also readily obtainable, yielding

$$
\frac{1}{h^{2}} \gamma_{2} \Lambda_{s t}^{2} v^{[0]}-D_{s t} \frac{\partial^{2} v^{[0]}}{\partial x_{1}^{2}}+\rho_{e} \frac{\partial^{2} v^{[0]}}{\partial t^{2}}=0 .
$$

We remark that $D_{s t}$ is the second order term in the expansion of the dispersion relation in the vicinity of thickness stretch resonance frequencies, see expansion Eq.(37). Moreover, if the general wave form solution Eq.(7) is inserted into Eq.(92) a dispersion relation is obtained that concurs with Eq.(37). It is then the case that the exact dispersion relation associated with the one dimensional approximate Eq. (92) is fully consistent with the approximation of the 2-dimensional problem Eq.(37).

\subsection{Shear resonance frequencies}

We proceed now with the derivation of an asymptotic model valid within the vicinity of thickness shear resonance frequencies. In order to derive the relative magnitudes of displacements, we expand all trigonometric functions in Eq.(13) and Eq.(14) for small $\eta$, and also make use of the expansions Eq.(39)-Eq.(42). The relative orders of displacements are given by $u_{2} \sim \eta u_{1}$. Thus we introduce

$$
u_{1}=l u_{1}^{*}, \quad u_{2}=l \eta u_{2}^{*},
$$

with the appropriate non-dimensional spatial and time variables given by

$$
x_{1}=l \xi, \quad x_{2}=\ln \zeta, \quad t=l \eta \sqrt{\frac{\rho_{e}}{\gamma_{2}}} \tau .
$$

We assume that in the vicinity of the shear resonance frequencies

$$
u_{1, \tau \tau}^{*}+\Lambda_{s h}^{2} u_{1}^{*} \sim \eta^{2} u_{1}^{*}, \quad k=1,2, \quad \Lambda_{s h}=\left(n-\frac{1}{2}\right) \frac{\pi}{2}, \quad n=1,2, \ldots .
$$


A system of governing equations for different orders may be obtained, given by

$$
\begin{aligned}
& \gamma_{2} u_{1, \zeta \zeta}^{(2 m)}+\gamma_{2} \Lambda_{s h}^{2} u_{1}^{(2 m)}+\alpha_{11} u_{1, \xi \xi}^{(2 m-2)}+\beta u_{2, \xi \zeta}^{(2 m-2)} \\
& -\gamma_{2} \eta^{-2}\left[u_{1, \tau \tau}^{(2 m-2)}+\Lambda_{s h}^{2} u_{1}^{(2 m-2)}\right]=0, \\
& \alpha_{22} u_{2, \zeta \zeta}^{(2 m)}+\beta u_{1, \xi \zeta}^{(2 m)}+\gamma_{2} \Lambda_{s h}^{2} u_{2}^{(2 m)}+\gamma_{1} u_{2, \xi \xi}^{(2 m-2)} \\
& -\gamma_{2} \eta^{-2}\left[u_{2, \tau \tau}^{(2 m-2)}+\Lambda_{s h}^{2} u_{2}^{(2 m-2)}\right]=0, \\
& \gamma_{2} u_{1, \zeta}^{(2 m)}+\left(\gamma_{2}-\sigma_{2}\right) u_{2, \xi}^{(2 m-2)}=0 \quad \text { at } \zeta=1 \text {, } \\
& \alpha_{22} u_{2, \zeta}^{(2 m)}+\alpha_{12} u_{1, \xi}^{(2 m)}=0 \quad \text { at } \quad \zeta=1 \text {, } \\
& u_{1}^{(2 m)}=u_{2}^{(2 m)}=0 \quad \text { at } \quad \zeta=-1 .
\end{aligned}
$$

6.2.1. Leading order problem. The leading order equations of motion and boundary conditions may be written as

$$
\begin{aligned}
& u_{1, \zeta \zeta}^{(0)}+\Lambda_{s h}^{2} u_{1}^{(0)}=0, \\
& \alpha_{22} u_{2, \zeta \zeta}^{(0)}+\beta u_{1, \xi \zeta}^{(0)}+\gamma_{2} \Lambda_{s h}^{2} u_{2}^{(0)}=0, \\
& u_{1, \zeta}^{(0)}=0, \quad \alpha_{12} u_{1, \xi}^{(0)}+\alpha_{22} u_{2, \zeta}^{(0)}=0 \quad \text { at } \zeta=1, \\
& u_{1}^{(0)}=u_{2}^{(0)}=0 \quad \text { at } \zeta=-1 .
\end{aligned}
$$

The solution of this problem is provided by

$$
\begin{aligned}
& u_{1}^{(0)}=v_{1}^{(0,0)}\left(\cos \left(\Lambda_{s h} \zeta\right)+\tan \left(\Lambda_{s h}\right) \sin \left(\Lambda_{s h} \zeta\right)\right), \\
& u_{2}^{(0)}=V_{2}^{(0,0)} \cos \left(\chi \Lambda_{s h} \zeta\right)+U_{2}^{(0,0)} \sin \left(\chi \Lambda_{s h} \zeta\right)+v_{2}^{(0,0)} \cos \left(\Lambda_{s h} \zeta\right)+u_{2}^{(0,0)} \sin \left(\Lambda_{s h} \zeta\right),
\end{aligned}
$$

where

$$
U_{2}^{(0,0)}=\frac{\cos \left(\Lambda_{s h} \chi\right) v_{1, \xi}^{(0,0)}}{\chi \alpha_{22} \Lambda_{s h}\left(\gamma_{2}-\alpha_{22}\right) \cos \left(2 \Lambda_{s h} \chi\right)}\left(-\frac{K_{2}}{\cos \left(\Lambda_{s h}\right)}+2 \beta \chi \alpha_{22} \sin \left(\Lambda_{s h}\right) \tan \left(\Lambda_{s h} \chi\right)\right),
$$

and

$$
\begin{aligned}
& V_{2}^{(0,0)}=\frac{1}{\cos \left(\chi \Lambda_{s h}\right)}\left[\sin \left(\chi \Lambda_{s h}\right) U_{2}^{(0,0)}+\frac{2 \beta \sin \left(\Lambda_{s h}\right) v_{1, \xi}^{(0,0)}}{\left(\gamma_{2}-\alpha_{22}\right) \Lambda_{s h}}\right], \\
& v_{2}^{(0,0)}=-\frac{\beta \tan \left(\Lambda_{s h}\right) v_{1, \xi}^{(0,0)}}{\Lambda_{s h}\left(\gamma_{2}-\alpha_{22}\right)}, \quad u_{2}^{(0,0)}=\frac{\beta v_{1, \xi}^{(0,0)}}{\Lambda_{s h}\left(\gamma_{2}-\alpha_{22}\right)} .
\end{aligned}
$$

The leading order displacement components have all been determined in terms of a single function, the long wave amplitude $v_{1}^{(0,0)}(\xi, \tau)$. An equation for $v_{1}^{(0,0)}$ will be derived by considering the next order problem.

6.2.2. Second order problem. A governing equation for $v_{1}^{(0,0)}$ may be found from the following second order equation of motion and boundary conditions

$$
\begin{aligned}
& \gamma_{2} u_{1, \zeta \zeta}^{(2)}+\gamma_{2} \Lambda_{s h}^{2} u_{1}^{(2)}=\gamma_{2} \eta^{-2}\left(\Lambda_{s h}^{2} u_{1}^{(0)}+u_{1, \tau \tau}^{(0)}\right)-\alpha_{11} u_{1, \xi \xi}^{(0)}-\beta u_{2, \xi \zeta}^{(0)}, \\
& \gamma_{2} u_{1, \zeta}^{(2)}+\left(\gamma_{2}-\sigma_{2}\right) u_{2, \xi}^{(0)}=0 \quad \text { at } \quad \zeta=1, \\
& u_{1}^{(2)}=0 \quad \text { at } \quad \zeta=-1 .
\end{aligned}
$$

The solution is given by

$$
\begin{aligned}
u_{1}^{(2)}=u_{1}^{(2,0)} \sin \left(\Lambda_{s h} \zeta\right)+v_{1}^{(2,0)} \cos \left(\Lambda_{s h} \zeta\right)+ & U_{1}^{(2,0)} \sin \left(\Lambda_{s h} \chi \zeta\right)+V_{1}^{(2,0)} \cos \left(\Lambda_{s h} \chi \zeta\right) \\
& +u_{1}^{(2,1)} \zeta \sin \left(\Lambda_{s h} \zeta\right)+v_{1}^{(2,1)} \zeta \cos \left(\Lambda_{s h} \zeta\right)
\end{aligned}
$$


where

$$
\begin{aligned}
& U_{1}^{(2,0)}=\frac{\beta V_{2, \xi}^{(0,0)}}{\chi \Lambda_{s h}\left(\alpha_{22}-\gamma_{2}\right)}, \quad V_{1}^{(2,0)}=-\frac{\beta U_{2, \xi}^{(0,0)}}{\chi \Lambda_{s h}\left(\alpha_{22}-\gamma_{2}\right)}, \\
& u_{1}^{(2,1)}=\frac{1}{2 \Lambda_{s h}}\left[\eta^{-2}\left(\Lambda_{s h}^{2} v_{1}^{(0,0)}+v_{1, \tau \tau}^{(0,0)}\right)+Q_{2} v_{1, \xi \xi}^{(0,0)}\right], \quad v_{1}^{(2,1)}=-\tan \left(\Lambda_{s h}\right) u_{1}^{(2,1)} .
\end{aligned}
$$

At this order, it is also possible to derive a governing equation for $v_{1}^{(0,0)}$ in the form

$$
D_{s h} v_{1, \xi \xi}^{(0,0)}-\gamma_{2} \eta^{-2}\left(\Lambda_{s h}^{2} v_{1}^{(0,0)}+v_{1, \tau \tau}^{(0,0)}\right)=0
$$

In terms of $x_{1}$ and $t$, and a new function $v^{[0]}\left(x_{1}, t\right)$, this equation is given by

$$
\frac{1}{h^{2}} \gamma_{2} \Lambda_{s h}^{2} v^{[0]}-D_{s h} \frac{\partial^{2} v^{[0]}}{\partial x_{1}^{2}}+\rho_{e} \frac{\partial^{2} v^{[0]}}{\partial t^{2}}=0 .
$$

We note that the second order correction term $D_{s h}$, see Eq.(43), occurs within the governing equation (115), again demonstrating asymptotic consistency.

\section{Concluding Remarks}

The problem of harmonic wave propagation in a compressible, pre-stressed elastic layer with one fixed and and free face has been investigated. Long and short wave approximations of the dispersion relation have been established. The long wave approximations have been used to establish the relative orders of displacement components within the vicinity of the thickness stretch and thickness shear resonance frequencies. In both cases, long wave leading order approximations of the displacement field have obtained in terms of the appropriate long wave amplitude. The leading order solution is formally completed through the derivation of a one-dimensional equation for the long wave amplitude; which is obtained from the second order problem. In each case, the dispersion relation associated with this one dimensional equation is consistent with the approximation previously obtained from the dispersion relation associated with the overarching two-dimensional problem. The lower dimensional theory is then demonstrated to be asymptotically consistent with the full higher dimensional theory.

\section{References}

[1] C. Liang, H. Lippmann, J. Najar, Effects of artificially induced vibrations on the prevention of coal mine bumps. Rockbursts and Seismicity in Mines 93: Proceedings of the 3rd international symposium, Kingston, Ontario, 1993.

[2] G.A. Holzapfel, T.C. Gasser, R.W. Ogden, A new constitutive framework for arterial wall mechanics and a comparative study of material models. Journal of elasticity 61 (2000) 1-48.

[3] G.A. Rogerson, Penetration of impact waves in a six-ply fibre composite laminate. Journal of sound and vibration 158 (1) (1992) 105-120.

[4] J.D. Kaplunov, D.G. Markushevich, Plane vibrations and radiation of an elastic layer lying on a liquid half-space. Wave Motion 17 (3) (1993) 199-211.

[5] J.D. Kaplunov, E.V. Nolde, Longwave vibrations of a nearly incompressible isotropic plate with fixed faces. The Quarterly Journal of Mechanics and Applied Mathematics 55 (3) (2002) 345-356.

[6] E.V. Nolde, G.A. Rogerson, Long wave asymptotic integration of the governing equations for a pre-stressed incompressible elastic layer with fixed faces. Wave motion 36 (2002) 287-304.

[7] R.W. Ogden, D.G. Roxburgh, The effect of pre-stress on the vibration and stability of elastic plates. International Journal of Engineering Science 31 (1993) 1611-1639.

[8] D.G. Roxburgh, R.W. Ogden, Stability and vibration of pre-stressed compressible elastic plates. International Journal of Engineering Science 32 (1994) 427-454.

[9] G.A. Rogerson, Some asymptotic expansions of the dispersion relation for an incompressible elastic plate. International Journal of Solids and Structures 34 (1997) 2785-2802. 
[10] E. Nolde, L. Prikazchikova, G. Rogerson, Dispersion of small amplitude waves in a pre-stressed compressible elastic plate. Journal of Elasticity 75(1) (2004) 1-29.

[11] A.V. Pichugin, G.A. Rogerson, An asymptotic membrane-like theory for long wave motion in a pre-stressed elastic plate. Proceedings of the Royal Society. Series A 458 (2002) 1447-1468.

[12] J.D. Kaplunov, E.V. Nolde, G.A. Rogerson, A low frequency model for dynamic motion in pre-stressed incompressible elastic structures. Proceedings of the Royal Society. Series A 456 2589-2610.

[13] J.D. Kaplunov, E.V. Nolde, G.A. Rogerson, An asymptotically consistent model for long wave high frequency motion in a pre-stressed elastic plate. Mathematics and Mechanics of Solids 7 (2002) 581-606.

[14] R. W. Ogden, Non-linear elastic deformations, Ellis Horwood, Chichester, 1984.

[15] J. D. Kaplunov, L. Yu. Kossovich, E. V. Nolde, Dynamics of thin walled elastic bodies, Academic Press, San Diego, 1998.

[16] M.A. Dowaikh, R.W. Ogden, On surface waves and deformations in a pre-stressed incompressible elastic solid. IMA Journal of Applied Mathematics 44 (1990) 261-284.

M.I. Lashhab

Faculty of Science, Elmergeb University, Libya

G.A. Rogerson

Department of Mathematics, Keele University, UK

e-mail: g.a.rogerson@keele.ac.uk

L.A. Prikazchikova

Department of Mathematics, Keele University, UK 